\title{
Estudo de Saúde Comparada: Os Modelos de Atenção Primária em Saúde no Brasil, Canadá e Cuba
}

\section{Comparative Health Study: Primary Health Care Models in Brazil, Canada and Cuba}

\author{
Joelma Cristina Santos' ${ }^{1}$, Walter Melo²
}

\begin{abstract}
Resumo
Os documentos governamentais e a literatura acadêmica apontam que, a partir da Constituição Federal de 1988, as políticas públicas de saúde do Brasil foram muito inspiradas nas políticas de bem-estar social de diversos países. Em relação à Estratégia Saúde da Família (ESF), destacam-se, principalmente, os trabalhos desenvolvidos no Canadá e em Cuba. Considerando-se que esses países têm estruturas políticas distintas e fatores sócio-histórico-culturais também bastante diversos, procurou-se investigar, com base em um estudo de saúde comparada, como tais características se efetivaram na constituição da ESF brasileira. Os dados indicam que as políticas de saúde cubanas têm muitas similaridades com as que são praticadas no Brasil, enquanto são percebidas diferenças entre a política de saúde canadense e a brasileira. Entende-se, portanto, que a ESF, no Brasil, se aproxima mais do modelo cubano de medicina familiar e que, apesar de constantemente citada como referência, a influência canadense, na prática, é bastante limitada.
\end{abstract}

Palavras-chave: Saúde Comparada. Atenção Primária em Saúde. Saúde da Família. Política de Saúde. Promoção da Saúde.

\begin{abstract}
Government documents and academic articles show that, since the Federal Constitution of 1988, public health programs in Brazil were strongly inspired by the welfare policies of several countries. In relation to the Family Health Strategy - FHS (ESF), the work done in Canada and Cuba stands out. Considering that these countries have different political structures and quite diverse socio-cultural-historical backgrounds, we sought to investigate, from a comparative perspective, how these characteristics are present in the Brazilian ESF. The obtained data shows us that the Cuban health policies have many similarities with the Brazilian, but there are policy differences between Canadian and Brazilian healthcare systems. Therefore, we consider that the Brazilian ESF is closer to the Cuban model of family healthcare and that, despite constantly cited as a reference, the Canadian influence is quite limited in practice.
\end{abstract}

Keywords: Comparative Health. Primary Health Care. Family Health Care. Health Policy. Health Promotion.

\footnotetext{
${ }^{1}$ Universidade Federal de São João del-Rei, São João del-Rei, Brasil. E-mail: joelma.psicologia@yahoo.com.br

${ }^{2}$ Universidade Federal de São João del-Rei, São João del-Rei, Brasil. E-mail: wmelojr@gmail.com
} 


\section{Introdução}

Para a Organização Mundial da Saúde (OMS), um sistema de saúde pode ser entendido como um conjunto de entidades responsáveis por intervenções na sociedade que têm a saúde como principal finalidade. Por se situarem num dado contexto político, econômico e técnico, e num determinado momento histórico, a definição, os limites e os objetivos dos sistemas de saúde nacionais são característicos de cada país. Todo sistema de saúde constitui-se em meio às forças políticas e às influências dos diversos grupos de interesse, refletindo valores sociais que são expressos nos limites jurídicos e institucionais das suas políticas (Opas, 2007). Assim, o modo como essa estruturação acontece determina as formas de funcionamento e organização dos sistemas de saúde, bem como a origem e o destino dos recursos que possibilitarão a prestação dos serviços de saúde. Políticas públicas constituem um conjunto de decisões que comunicam meios, estratégias, regras e finalidades para as tomadas de decisões na legislação e na administração pública, podendo se originar em todos os níveis de um governo. Do mesmo modo que os sistemas, a qualidade das políticas de saúde está limitada pela história, cultura, política, economia e pelos fundamentos sociais dos contextos nos quais se aplicam (Opas, 2007). As políticas de saúde são importantes porque afetam direta ou indiretamente todas as dimensões da vida cotidiana, abrangendo comportamentos e ações que contribuem para a melhoria do estado de saúde, incluindo as atividades econômicas e produtivas a ela relacionadas.

Nesse contexto, a análise comparada em saúde, embora ainda se caracterize como um campo em desenvolvimento, pode oferecer contribuições relevantes para a organização e a prestação dos serviços em saúde. Os objetivos dos estudos nessa área consistem, principalmente, em conhecer determinações, causalidades e inter-relações entre sistemas e serviços de saúde, com base em semelhanças, diferenças ou arranjos entre elementos que podem ou não ser contemporâneos e/ou ocorrer em espaços distintos (Conill, Mendonça, Silva \& Gawryszewski, 1991; Conill, 2007; 2011). A possibilidade de avaliar a influência de variáveis históricas, culturais e políticoadministrativas possibilita ampliar o campo de análise das relações entre a elaboração de políticas de saúde e a efetiva concretização destas por meio da prestação de serviços, considerando, assim, o contexto em que estas ocorrem. Nesse sentido, a análise comparada pode ser um valioso instrumento para que se repense as concepções, os serviços e as práticas em saúde, tendo em vista que comparar não se resume a estabelecer padrões nem a recomendar a cópia ou a transferência de experiências, mas a encontrar referências para a busca de novos caminhos (Conill et al., 1991).

Conforme Labra (2001), após a Segunda Guerra Mundial, acreditou-se que ocorreria um desenvolvimento progressivo dos sistemas de saúde devido à força da urbanização, da industrialização, do crescimento econômico, dos avanços científicos e tecnológicos, bem como da 
expansão do estado de bem-estar social. No entanto, o que se percebeu é que a evolução dos serviços de saúde no mundo não se submeteu a um imperativo sequencial, predominando, muitas vezes, uma combinação de fatores influentes $\mathrm{o}$ suficiente para determinar a natureza dos sistemas de saúde. Sendo assim, entende-se que diversos aspectos, como semelhanças entre países, variáveis históricas e forças políticas, podem contribuir para que um país adote uma ou outra configuração, que resulta sempre num caso único.

Considerando-se que os sistemas, serviços e políticas de saúde são fenômenos complexos, é preciso assinalar que certos entraves conceituais e metodológicos ainda persistem nos estudos de análise comparada. Entre estes se destacam a concepção de saúde, a análise das determinações do setor, o discernimento entre aspectos referentes aos sistemas e às políticas de saúde, os métodos e técnicas de avaliação, a ausência de dados homogêneos de um sistema de saúde em relação a outro e a escolha entre indicadores, o que pode tanto limitar a comparação como prejudicar o aprimoramento das análises (Conill et al., 1991; Hortale, Conill e Pedroza, 1999). Hortale et al. (1999) ressaltam que essas limitações teóricometodológicas são alguns dos desafios presentes na construção de um modelo efetivo para a análise comparada da organização de serviços de saúde. Assim, o desafio que se apresenta é de, ao mesmo tempo, efetuar um recorte no objeto que possibilite a comparação, mas que esteja relacionado a um contexto social, histórico, econômico e político (Conill, 2011).
Exemplos da diversidade e das dificuldades presentes na área podem ser ilustrados pelos estudos de Labra (2001), Santos e Ugá (2007), Gerschman (2008), Rabello (2010) e Bonet (2014). Labra (2001) comparou aspectos político-institucionais das transformações dos sistemas de saúde do Chile e do Brasil, implantadas no último século, enfatizando as "modernizações" neoliberais chilenas do início da década de 1980, durante a ditadura de Pinochet, e as reformas empreendidas no Brasil, em fins da mesma década, no processo de redemocratização. O objetivo do estudo de Santos e Ugá (2007) consistiu em analisar os processos de reforma dos sistemas de saúde na Argentina, no Brasil, na Colômbia e no México, dentro do contexto político-econômico da década de 1980, considerando a relação entre descentralização e território. Gerschman (2008) comparou a relação entre público e privado no contexto dos sistemas de saúde universais a fim de demonstrar semelhanças e diferenças entre as políticas de saúde do Brasil e da União Europeia. Como a União Europeia é formada por vários países, enquanto o Brasil é um só país, a autora argumenta que seu estudo pode ser considerado "um exercício teórico-conceitual", pois adotou "uma licença metodológica" (p. 1442). Rabelo (2006; 2010), por sua vez, faz análises comparativas entre Brasil e Canadá a partir da promoção da saúde considerando quatro categorias - gestão, intersetorialidade, qualidade de vida e participação social -, e conclui que a promoção da saúde ainda não estava consolidada nos dois países, predominando o modelo flexeneriano. A partir da noção médicos da pessoa, 
Bonet (2014) implementou um estudo antropológico sobre a atenção primária em saúde na Argentina (Buenos Aires e região) e no Brasil, mais especificamente em Niterói/RJ, que adaptou o modelo de medicina da família de Cuba. Nesse sentido, a polissemia do termo medicina da família deve-se à adequação ao contexto em que é praticada, configurando uma “epistemologia sincrética, que se atualiza nos sujeitos" (p. 254).

Este artigo se insere no contexto da análise comparada em saúde, reconhecendo as dificuldades inerentes ao processo de comparação, mas, ainda assim, buscando contribuir para uma melhor compreensão sobre a constituição do sistema de saúde brasileiro, notadamente da atenção primária. A estrutura organizacional e as diretrizes sanitárias do Sistema Único de Saúde (SUS), no Brasil, foram inspiradas, em grande parte, nas políticas de bem-estar social de vários países (Aguiar, 1998; Albuquerque \& Melo, 2010; Bonet, 2014; Conill, 2008; Viana \& Dal Poz, 2005; Silveira, 2008; entre outros). A partir da promulgação da Constituição Federal de 1988, Inglaterra, Suécia, Portugal, Itália, Canadá, Cuba, entre outros países, serviram de referência para a construção das políticas públicas de saúde brasileiras.

Em relação à Estratégia Saúde da Família (ESF), as principais diretrizes formuladas foram baseadas em modelos de assistência à família desenvolvidos em outros países e que serviram de guias para a elaboração do programa brasileiro. Como o Brasil apresenta características próprias, a ESF é uma adaptação de várias experiências internacionais, entre as quais examinaremos, neste artigo, os trabalhos desenvolvidos no Canadá e em Cuba, países reconhecidos por desenvolverem estratégias de atenção primária e enfatizarem a promoção da saúde. Além do fato de esses países terem servido de inspiração para as políticas brasileiras, a opção por Canadá e Cuba, para esta análise, deve-se ao fato de serem tidos como referência em qualidade na prestação de serviços de saúde, alcançando alguns dos melhores indicadores, no continente americano, segundo dados da OMS (2015). Além disso, justifica-se a necessidade de uma análise mais aprofundada dos dois países, uma vez que, apesar de produzirem resultados semelhantes em saúde, Canadá e Cuba apresentam diferenças importantes entre si, do ponto de vista cultural, social, político e econômico que, consequentemente, interferem na estruturação de seus sistemas de saúde.

Em relação ao Brasil, poder-se-ia pensar que as semelhanças que o país tem com o Canadá, como grande extensão territorial e mesmo sistema econômico - capitalismo -, poderiam ter influência decisiva sobre a organização dos serviços, pelos efeitos gerados na acessibilidade geográfica aos serviços e no financiamento, por exemplo. Por outro lado, também é possível pensar que fatores sóciohistóricos de Cuba - colonização de exploração, cultura latina, contexto de desigualdades sociais - poderiam predominar no delineamento dos objetivos e características que o sistema brasileiro teria. Desse modo, o objetivo deste trabalho consiste em avaliar em que medida as características apresentadas pela atenção primária cubana e canadense se combinaram e se 
concretizaram na constituição da ESF no Brasil.

O presente artigo é o resultado de uma pesquisa bibliográfica e, como assinala Gil (1999), esse tipo de pesquisa é desenvolvido com base em material já elaborado, principalmente livros e artigos científicos, além de publicações avulsas, boletins, jornais, pesquisas, monografias, dissertações, teses, entre outros. Dessa forma, buscou-se levantar e analisar materiais que retratassem os sistemas de saúde do Canadá, de Cuba e do Brasil a fim de averiguar contribuições teóricas e práticas das políticas públicas canadenses e cubanas à estruturação do sistema e dos serviços de saúde na atenção primária no Brasil. De modo geral, a base de dados da pesquisa foi constituída por artigos científicos, disponíveis em bases eletrônicas de dados gratuitas e comumente reconhecidas - SciELO e Portal de Periódicos Capes -, sendo os artigos localizados por meio das palavras-chave "sistema de saúde cubano", "sistema de saúde canadense", "sistema de saúde brasileiro", "Cuba" e "Canadá". Especificamente, para a descrição do modelo brasileiro de assistência à saúde, foram utilizados também leis, portarias e documentos oficiais do Ministério da Saúde. Para o levantamento de dados acerca do modelo de assistência à saúde do Canadá, foram usados ainda documentos oficiais disponibilizados nos sites dos governos federal e da província de Quebec, considerada a que tem o melhor sistema de saúde do país e também a principal referência canadense para o sistema brasileiro (Conill, 2000; Viana \& Dal Poz, 2005). No caso de Cuba, também foram utilizadas informações disponíveis em sites de embaixadas do país e, devido a dificuldades de acesso a documentos oficiais do governo de Cuba, foram feitas ainda buscas por artigos científicos de autores cubanos, no SciELO e no Portal de Periódicos Capes, por meio das palavras-chave "sistema salud cuba" e "atención primaria". Além dessas fontes, foram utilizados, também, como material-base, livros de referência no campo da saúde coletiva, teses de doutorado e dissertações de mestrado e documentos da Organização PanAmericana de Saúde, acessados por meio de site de buscas e utilizando as mesmas palavraschaves usadas no levantamento dos artigos científicos.

$$
\text { Com o objetivo de facilitar a }
$$
identificação e a visualização do que será comparado entre os modelos de atenção à saúde do Brasil, do Canadá e de Cuba, foram definidos os seguintes parâmetros para análise: a) princípios do sistema de saúde, b) organização do sistema de saúde, c) relação público-privado e d) cobertura a medicamentos. Acredita-se que a escolha por essas categorias proporcionou uma visão geral dos modelos de atenção primária à saúde dos três países, permitindo a verificação das principais semelhanças, discrepâncias e influências na estruturação dos serviços de saúde brasileiros. Este artigo está centrado na atenção primária em saúde por considerar que a discussão dessa temática é vital ao se estudar a organização do sistema de saúde brasileiro. A seguir, são apresentados um brevíssimo histórico acerca da constituição dos sistemas de saúde brasileiro, canadense e cubano e as principais características de cada um para, em seguida, serem comparados de acordo com os 
parâmetros de análise definidos.

\section{As Principais Características da ESF}

\section{Brasileira}

\section{Princípios do Sistema de Saúde}

A partir da década de 1980, a Reforma Sanitária teve por objetivo realizar uma ampla transformação do sistema de saúde e contribuiu de maneira decisiva para a reformulação das políticas de saúde brasileiras. Tal processo culminou com a Constituição Federal de 1988, que estabeleceu o SUS, legitimado por meio da Lei $\mathrm{n}^{\circ} 8.080 / 90$, e reafirmou a "saúde como direito de todos e dever do Estado". Fundamentado na premissa de que a produção de saúde é determinada socialmente, o SUS foi instituído como estratégia para garantir atendimento integral e cobertura universal, de forma descentralizada (Costa, Pontes \& Rocha, 2006). A criação do SUS preconizou as condições para a promoção, a proteção e a recuperação da saúde, e incorporou, no art. 198 da Constituição Federal, diretrizes que devem obedecer a 13 princípios, expressos no art. $7^{\circ} \mathrm{da}$ Lei no 8.080/90.

- Universalidade: todos os indivíduos têm direito à saúde, em todos os níveis de assistência, por meio de serviços de saúde e políticas econômicas e sociais.

- Integralidade: assistência curativa aliada à prevenção de doenças e à promoção da saúde, o que envolve todos os níveis de complexidade do sistema.

- Autonomia: toda pessoa deve ter sua autonomia preservada na defesa da sua integridade física e moral.
- Equidade: todo cidadão é igual perante o SUS, sem privilégios de qualquer espécie, devendo ser atendido segundo as suas necessidades.

- Informação: toda pessoa assistida tem direito à informação sobre sua saúde.

- Divulgação sobre os serviços: as informações sobre o potencial dos serviços de saúde e a sua utilização pelos usuários devem ser divulgadas.

- Utilização da Epidemiologia: as informações epidemiológicas devem ser usadas a fim de que se estabeleçam prioridades, se aloquem recursos e se realize orientação programática.

- Participação Popular gestão participativa das políticas de saúde, por meio das Conferências e Conselhos de Saúde.

- Descentralização: os serviços de saúde estão estruturados de acordo com os níveis de administração pública, com ênfase na esfera local e abrangendo os princípios da Regionalização - os serviços de saúde estão organizados conforme a sua especificidade - e da Hierarquização - os serviços de saúde são organizados segundo a sua complexidade.

- Integração: as ações de saúde, meio ambiente e saneamento básico estão integradas em nível executivo.

- Conjugação de Recursos: os recursos financeiros, tecnológicos, materiais e humanos da União, dos Estados, do Distrito Federal e dos Municípios estão conjugados na prestação dos serviços de assistência à saúde.

- Resolubilidade: capacidade de resolução dos serviços em todos os níveis de assistência. 
- Organização dos serviços: os serviços públicos devem estar organizados, de modo a evitar duplicidade de meios para os mesmos fins.

\section{Organização do Sistema de Saúde}

A partir da década de 1990, a família e a comunidade adquirem mais importância para a reorganização da assistência à saúde e algumas estratégias, focadas na prevenção de doenças e na promoção da saúde na esfera comunitária, são implantadas. Em 1991, é criado o Programa de Agentes Comunitários de Saúde (Pacs) visando diminuir as mortalidades infantil e materna, por meio do acesso a serviços de saúde nas regiões mais pobres do país (Viana \& Dal Poz, 2005). A ampliação do Pacs se deu, em 1994, com a criação, do Programa Saúde da Família (PSF), que promoveu a valorização do território, o estabelecimento de vínculos estreitos com a população, o trabalho em equipes multidisciplinares, a promoção da saúde por meio de ações intersetoriais e o estímulo à participação comunitária, entre outros aspectos. Após avançar em todas as regiões do país, o PSF reorienta o modelo assistencial, fundamentandoo na atenção básica e constituindo-se como eixo ordenador da saúde no Brasil.

Ao se tornar o braço auxiliar na implantação do SUS e na organização dos sistemas locais de saúde, o PSF deixa de ser um Programa para ser definido como Estratégia Saúde da Família (Viana \& Dal Poz, 2005). Na ESF, a Unidade de Saúde da Família está inserida no nível primário de ações e serviços do sistema local de saúde, caracterizando-se por ser o contato preferencial dos usuários e a principal porta de entrada do sistema de saúde. Pelos princípios da integralidade e da hierarquização, a Unidade deve assegurar a referência e a contrarreferência de informações em saúde para os demais níveis do sistema (secundário ou terciário), sempre que se fizer necessária uma maior complexidade tecnológica.

A Portaria $n^{\circ} 2.488$, de 2011, estabelece que a Equipe de Saúde da Família deve ser multiprofissional e composta por, no mínimo, médico generalista ou médico de família, enfermeiro, auxiliar de enfermagem e agentes comunitários de saúde, em número suficiente para cobrir toda a população cadastrada. Cada agente deve ser responsável por, no máximo, 750 pessoas, a fim de realizar cadastramentos domiciliares, ações educativas, atividades de promoção da saúde e de prevenção de doenças, entre outras atribuições. As Equipes devem ter responsabilidade sanitária pelos seus territórios de referência e as suas funções são distribuídas entre visitas domiciliares, ações programáticas e atendimentos no consultório pelo médico e pela enfermeira. Além disso, a Portaria no 2.488/11 determina que o trabalho deve ser desenvolvido com o suporte dos Núcleos de Apoio de Saúde da Família (Nasf), criados com a finalidade de aumentar a abrangência e a eficácia das iniciativas de atenção primária. As equipes do Nasf são formadas por profissionais de nível superior de diferentes áreas e atuam sobre demandas identificadas pelas Equipes Básicas de Saúde, mas não estão organizados como serviços com unidades físicas independentes. As ações de apoio desenvolvidas pelo Nasf abrangem discussões de casos, construção de 
projetos terapêuticos, educação permanente, ações intersetoriais, atividades de prevenção e promoção da saúde, discussão do processo de trabalho das equipes, entre outras tarefas.

De acordo com a Portaria $n^{\circ} 648$, de 2006, as Equipes de Saúde Bucal trabalham integradas a uma ou duas Equipes de Saúde da Família, com responsabilidade sanitária pela mesma população e território, aos quais está vinculada. As Equipes de Saúde Bucal devem ter, basicamente, cirurgião dentista e auxiliar de consultório dentário, sendo que, em casos específicos, pode ter técnico de higiene dental.

\section{Relação Público-Privado}

No Brasil, o setor privado da saúde antecede historicamente a constituição do SUS. Conforme o art. 199 da Constituição Federal de 1988, as instituições privadas podem participar do SUS, de forma complementar, seguindo as diretrizes deste. Mais tarde, a fim de regulamentar a atuação dos planos privados de saúde, a Lei no 9.565, de 1998, criou a Agência Nacional de Saúde Suplementar (ANS), que deve controlar, avaliar e fiscalizar os procedimentos dos serviços privados de saúde. No Brasil, a iniciativa privada tem liberdade para dar cobertura aos mesmos serviços de saúde cobertos pelo SUS e, na prática, têm grande abrangência, principalmente, no que se refere à atenção ambulatorial. A Lei no 8.080/90 também estabelece que o SUS pode celebrar convênios ou contratos de direito público com a iniciativa privada, a fim de garantir a cobertura assistencial à população de uma determinada área, quando as disponibilidades do SUS forem consideradas insuficientes. Nesses casos, as entidades filantrópicas e as entidades sem fins lucrativos têm preferência.

\section{Cobertura a Medicamentos}

A Política Nacional de Medicamentos do Brasil (Ministério da Saúde, 2001), aprovada pela Lei $\mathrm{n}^{\circ} 3.916 / 98$, assegura o acesso gratuito à medicação necessária a pacientes atendidos no SUS, em todos os níveis de atenção. Conforme o art. $6^{\circ}$ da Lei $n^{\circ} 8.080 / 90$, o fornecimento de medicamentos atende ao princípio da integralidade da assistência, pois o medicamento não é concebido isoladamente, mas é um dos componentes do tratamento. Os medicamentos considerados essenciais à terapêutica da maioria dos problemas de saúde são ofertados a baixo custo aos pacientes atendidos na rede privada de saúde, por meio do Programa Farmácia Popular do Brasil. Criado em 2004 pelo Decreto no 5.090 e atualmente regulado pela Portaria n ${ }^{\circ} 111 / 2016$, o Programa Farmácia Popular do Brasil, disponibiliza medicamentos, via convênios entre Estados, Distrito Federal, Municípios e hospitais filantrópicos, além de farmácias e drogarias privadas, onde o preço dos medicamentos é subsidiado.

\section{As Principais Características do Sistema de Saúde Canadense \\ Princípios do Sistema de Saúde}

Desde a década de 1970, o sistema de saúde canadense se caracteriza, predominantemente, por ser público, fornecendo cobertura universal e abrangente. As dez províncias e os três territórios do país 
compõem treze planos de saúde distintos, mas entrelaçados pelos princípios norteadores ditados pelo governo federal, garantindo, assim, mais semelhanças do que diferenças entre os planos (Conill, 2000). As províncias e territórios canadenses devem satisfazer cinco princípios (em termos administrativos, de cobertura e de organização) a fim de se qualificarem para receber as transferências federais completas sobre saúde (Health System and Policy Division, 1999).

- Administração pública: a administração dos planos de seguro de saúde das províncias e dos territórios deve ser realizada em base não lucrativa por uma autoridade pública, identificada pelo governo provincial ou territorial.

- Abrangência: são segurados todos os serviços clinicamente necessários prestados por médicos e hospitais. Os serviços hospitalares segurados incluem o tratamento dos doentes internados em enfermarias (a não ser que seja clinicamente necessário o alojamento em quartos privados ou semiprivados), os medicamentos, os suprimentos e as análises de diagnose, bem como uma ampla gama de serviços hospitalares a pacientes ambulatoriais.

- Universalidade: todos os residentes registrados no sistema de saúde de uma província ou território são cobertos pelo segurosaúde em termos e condições uniformes. Novos moradores (vindos de outra província ou território) ou imigrantes têm direito ao plano, mas estão sujeitos a uma carência de, no máximo, três meses.

- Acessibilidade: todos os residentes de uma província ou território devem ter acesso aos serviços hospitalares, médicos e cirúrgicodentários e não podem ter seu atendimento impedido por nenhum tipo de barreira, como pagamento de taxas, discriminação de raça, idade, sexo, estado de saúde ou qualquer outra circunstância.

\section{Transferibilidade on Portabilidade:}

residentes que se transferem de uma província para outra ou mesmo para fora do país, sob determinado período e por razões previamente acordadas e estabelecidas, têm cobertura garantida de serviços segurados e mesmo de alguns serviços não segurados, a depender da província de origem.

\section{Organização do Sistema de Saúde}

O Canadá não tem médicos empregados pelo governo federal, sendo que a maioria trabalha em consultórios privados. Quando necessitam de assistência médica, os canadenses dirigem-se, geralmente, ao médico ou clínica da sua preferência, onde apresentam o cartão de seguro de saúde emitido a todos os residentes admissíveis de cada província/território. Esses médicos são remunerados por serviços prestados, após apresentarem suas notas de honorários diretamente ao plano de seguro de saúde provincial/territorial para pagamento. As remunerações dos serviços dos médicos são negociadas entre as províncias e as associações médicas provinciais/territoriais, estabelecidas, em geral, por períodos de dois a quatro anos (Sório, 2006). Assim, os canadenses não pagam diretamente pelos serviços segurados de médicos e hospitais, não existindo taxas 
dedutíveis, pagamentos complementares ou limites financeiros na cobertura dos serviços segurados. Entretanto, existem pagamentos praticados por pacientes dispostos a arcar com o custo total dos serviços aos poucos médicos cuja prática é totalmente externa ao sistema público. Alguns médicos trabalham em centros de saúde comunitários, clínicas hospitalares ou serviços ambulatoriais em hospitais (Health System and Policy Division, 1999). Os dentistas trabalham de maneira independente do sistema de assistência médica, exceto quando há necessidade de cirurgia dental hospitalar (o que é coberto pelo governo).

Os médicos de assistência primária (como os generalistas, que representam cerca de 51\% dos médicos ativos no país) estabelecem, na maioria das vezes, o contato inicial com o sistema de assistência médica convencional, controlando o acesso a maior parte dos especialistas, dos profissionais afins, das admissões hospitalares, das análises diagnósticas e da administração de medicamentos (Sório, 2006). Noventa e cinco por cento dos hospitais canadenses atuam como entidades privadas sem fins lucrativos e são administrados por conselhos de dirigentes comunitários, organizações filantrópicas ou autarquias municipais. O setor hospitalar privado compreende, principalmente, os estabelecimentos de cuidados em longo prazo ou os serviços especializados, tais como centros de desintoxicação (Health System and Policy Division, 1999).

De acordo com Marchildon (2013), a efetiva descentralização do sistema de saúde canadense se deve a três fatores principais: 1 . O financiamento e a prestação de serviços são de responsabilidade das províncias e dos territórios. 2. O fato de os médicos não serem funcionários do governo. 3. A presença de autoridades de saúde regionais (ASR), que fazem a planificação detalhada dos serviços de saúde para populações definidas. No entanto, o autor indica que, nos últimos anos, ocorreu uma diminuição das ASR, apontando para uma tendência à centralização. Mas, como assinala Sório (2006), o sistema de saúde canadense, comparado a outros, é considerado um dos mais descentralizados do mundo, uma vez que as províncias e territórios têm grande autonomia para desenvolver seus sistemas locais, sendo que cada província/território tem seu próprio Ministério da Saúde. A gestão e a prestação dos serviços de saúde cabem a cada província/território, que estabelece as prioridades, financia e aloca os recursos entre as regiões, concede licenças, cadastra e paga os profissionais. Além disso, destina recursos financeiros para hospitais, controla custos e avalia a prestação da assistência médica, de serviços correlatos e certos aspectos da saúde pública. As províncias e territórios também prestam benefícios que vão além dos cobertos pelo governo federal, como clínica dentária, oftalmologia, serviços de outros profissionais de saúde, medicamentos, cuidados domiciliares (bome care) e reabilitação. Os governos municipais têm um papel mais limitado, exercendo funções de vigilância de estabelecimentos de alimentação, imunizações e serviços de ambulância (Sório, 2006).

Como exemplo de sistema de saúde provincial, pode-se citar o caso do Quebec, 
considerado referência em aplicação de política de saúde com ênfase na comunidade, geograficamente definida e com ações que se destacam pelo caráter inovador (Conill, 2000). O modelo de assistência à saúde do Quebec está hierarquizado em três níveis, sendo que os estabelecimentos, dispostos em redes, operam em complementaridade: no nível central, situase o Ministério da Saúde, que atua no planejamento, financiamento, acompanhamento e avaliação das políticas sócio-sanitárias; no nível regional, estão as Agências da Saúde e os Serviços Sociais (ASSS), responsáveis pela coordenação nos respectivos territórios; e no nível local, o Centro de Saúde e de Serviços Sociais (CSSS) constituiu a base da prestação de serviços, especialmente os de média e de alta complexidade, com a finalidade de garantir acesso, atendimento, acompanhamento e coordenação dos serviços designados a uma população definida territorialmente (Gouvernement du Québec, 2008).

Assim como em todo o Canadá, os clínicos gerais e os especialistas do Quebec são considerados profissionais liberais. No entanto, a grande maioria exerce a sua profissão exclusivamente no sistema de saúde pública da província. Entre os diversos tipos de práticas, podem ser citados os Grupos de Medicina de Família, constituídos por profissionais que atendem às populações cadastradas, em estreita colaboração com enfermeiras da rede de saúde pública (Conill, 2008). No âmbito das clínicas privadas, os Grupos de Medicina de Família respondem por uma lista de pacientes e prestam atendimento domiciliar e fora de horário, recebendo subsídio governamental para infraestrutura e pessoal (Conill, 2009).

\section{Relação Público-Privado}

No Canadá, existem serviços de saúde suplementares que são fornecidos principalmente pelo setor privado, devendo ser custeados pela população. Os indivíduos podem optar pelo seguro privado ou beneficiar-se de um plano de seguro empresarial (vinculado ao emprego), que lhes paga determinada parcela das despesas dos serviços de saúde suplementares (Health System and Policy Division, 1999). Os seguros de saúde privados não podem, por lei, concorrer na faixa de serviços disponíveis na rede pública, mas podem competir no mercado dos benefícios suplementares para quartos privados, despesas com medicamentos, cirurgias estéticas, cuidados domiciliares e tratamentos dentários. Sório (2006) argumenta que a amplitude da ação estatal na oferta de serviços de saúde leva o sistema canadense a ser, efetivamente, o único em que a palavra suplementar é realmente aplicável para a participação privada no conjunto da assistência à saúde da população. Isso porque o segmento privado é proeminente apenas em ações que não são consideradas de grande relevância para as autoridades sanitárias.

\section{Cobertura a Medicamentos}

A assistência farmacêutica é realizada pelas províncias e territórios, que custeiam integralmente os hemoderivados, as vacinas e os medicamentos ministrados nos hospitais e ambulatórios (Rêgo, 2000). Os medicamentos 
prescritos para uso fora do ambiente hospitalar são parcialmente cobertos pelo sistema público, no entanto, a extensão da cobertura de medicamentos varia muito entre as províncias e os territórios, sendo que alguns planos cobrem grande parte das prescrições farmacêuticas, enquanto outros são mais focados em determinados grupos, como idosos e desempregados. Além disso, anualmente, as províncias definem, segundo critérios próprios, listas de medicamentos passíveis de cobertura, sendo que algumas províncias estabelecem um teto de cobertura e outras adotam o pagamento conjunto que, em parte, podem ser deduzidos do imposto de renda. Em média, cerca de 50\% das necessidades de medicamentos da população são atendidas pelos seguros públicos, enquanto o restante é coberto por seguros privados ou pelos usuários (Rêgo, 2000). O Governo Federal cobre os custos de medicamentos das nações aborígenes, das forças armadas e dos veteranos de guerra (Health System and Policy Division, 1999).

\section{As Principais Características do Sistema de}

\section{Saúde Cubano}

\section{Princípios do Sistema de Saúde}

O sistema de saúde em Cuba passou por profundas mudanças, desde a revolução socialista de 1959, quando a saúde foi reconhecida como um direito do povo e um dever e responsabilidade do Estado. Desde então, a atenção médica ambulatorial do país passou por três modelos: o policlínico integral (1964-1974), o policlínico comunitário (19741984) e o modelo cubano de medicina familiar (desde 1984). Esses modelos se sucederam em função do desenvolvimento social e das mudanças no quadro epidemiológico do país (de uma predominância de doenças infectocontagiosas para uma maior prevalência de enfermidades crônicas não transmissíveis), além das próprias demandas advindas da prática (Soberats, Márquez e Galbán, 2011). O Sistema Nacional de Saúde (SNS) de Cuba é único, descentralizado, com financiamento totalmente público e orientado por sete princípios.

- Caráter estatal e social da medicina: os serviços de saúde constituem um direito de todos os cidadãos e estão organizados em um único sistema, sob a responsabilidade do Estado, e regido por um conjunto de disposições jurídicas.

- Orientação profilática: a prestação dos serviços está voltada para a intervenção sobre os fatores determinantes da saúde coletiva, abrangendo desde melhorias sanitárias até a identificação de fatores de risco em pessoas vulneráveis, a fim de se oferecer proteção individual.

\section{- Acessibilidade e gratuidade geral: os} serviços de saúde devem ser acessíveis geograficamente e oferecidos de forma gratuita a toda a população.

- Integralidade e desenvolvimento planejado: os serviços de saúde são de caráter integral, devem ter alta qualificação e cobrir toda a população de maneira equitativa. O planejamento em saúde é um processo único e centralizado, visando ao desenvolvimento igualitário da prestação de serviços em todo o país, com ênfase nas províncias mais atrasadas em relação à capital. 


\section{- Unidade entre ciência, docência e prática}

médicas: o ensino superior em Medicina está subordinado ao Ministério de Saúde Pública (Minsap), que assume a responsabilidade pela formação e educação continuada dos profissionais de níveis técnico e superior. Partindo da premissa de se combinar o ensino e a atuação profissional, os estudantes se desenvolvem por meio do trabalho direto nas unidades de saúde, sob a supervisão dos docentes que também atuam nas unidades. Entre as demandas do trabalho docente dos profissionais nas unidades de saúde, as atividades de pesquisa também são privilegiadas.

- Participação da população: os serviços de saúde devem ser desenvolvidos com a participação ativa da comunidade organizada, que tem papel importante no acesso à informação e no controle das atividades.

- Internacionalismo: este princípio se efetiva de duas formas: (i) por meio da graduação em Medicina de estudantes estrangeiros que vão para Cuba e (ii) pelo envio de profissionais cubanos para outros países a fim de prestar serviços de assistência médica (como o ocorrido recentemente, no Brasil, por meio do Programa Mais Médicos, instituído pela Lei no 12.871/13).

\section{Organização do Sistema de Saúde}

No nível nacional do SNS cubano, situase o Minsap, que exerce as funções normativas de coordenação e controle, subordinado ao Estado e ao Governo da República, que mantém uma hierarquia técnica sobre as direções provinciais e municipais de saúde. No SNS existem três níveis de atenção médica: primário, secundário e terciário, que estão interrelacionados conforme a localização territorial e o seu grau de complexidade, seguindo o princípio da acessibilidade geográfica dos serviços (Soberats et al., 2011).

A atenção médica primária constitui a porta de entrada do SNS e compreende o conjunto de atividades e procedimentos que visam a garantir a saúde de uma determinada comunidade, tendo por base o enfoque clínico, epidemiológico e social dos problemas de saúde da família (Aguiar, 1998). O Consultório Médico da Família (CMF), onde atuam o médico e a enfermeira da família, está situado nesse nível, constituindo-se como a célula básica do Policlínico (nas áreas urbanas) e dos Hospitais Rurais (nas áreas não urbanas), compondo um contínuo que articula os níveis secundário e terciário, mediante o sistema de referência e contrarreferência de informações em saúde. Os CMFs podem ser de dois tipos: um que abrange mais de um consultório, com acomodações para o médico e enfermeira, e outro - mais comum com acomodação apenas para o médico. Todas as áreas de saúde estão vinculadas a um serviço odontológico, sendo que cada dentista se vincula a dois Consultórios Médicos de Família. Nos lugares onde isso não é possível, por não se dispor de recursos humanos suficientes, vinculase um dentista a um número determinado de CMF.

Todos os CMFs estão atrelados a um Policlínico ou Hospital Rural, sendo que o número de CMFs vinculados a cada Policlínico/Hospital Rural depende da extensão e das características do território em que está 
localizado, da população compreendida e da presença de centros laborais, educacionais ou outros, em seus limites geográficos. Os Policlínicos e Hospitais Rurais caracterizam-se como centros integradores e coordenadores dos serviços de saúde, de administração, de docência e de pesquisa em cada território definido, estando vinculados a hospitais clínico-cirúrgico, pediátrico e ginecológico-obstétrico, em correspondência à regionalização dos serviços. Essas instituições se denominam Hospitais-Base e estão relacionadas às instituições de nível de atenção terciário, a fim de viabilizar consultas externas de várias especialidades (Soberats et al., 2011).

Cada Policlínico conta com serviços de urgência, meios diagnósticos, consultas externas de medicina integral, medicina interna, pediatria, obstetrícia e ginecologia, e equipe multidisciplinar de atenção gerontológica. Além disso, são efetuadas consultas externas de especialidades não básicas, oferecidas por profissionais provenientes dos Hospitais-Base, nas áreas de saúde mental, enfermagem, assistência social, podologia, medicina natural e tradicional, além de serviços especializados de planejamento familiar (Soberats et al., 2011).

O médico e a enfermeira da família formam a Equipe Básica de Saúde e constituem o principal elo para a realização de ações de promoção da saúde e prevenção de doenças, buscando atuar de forma integral, regionalizada e com a participação ativa da comunidade situada em seu entorno (Aguiar, 1998). Cada conjunto de 15 a 20 Equipes Básicas de Saúde, acompanhado de especialistas em medicina integral (chefe), pediatra, ginecologista-obstetra, dentista, psicólogo, supervisor de enfermagem e assistente social compõem uma equipe multidisciplinar, denominada Grupo Básico de Trabalho (GBT). Os especialistas dos GBTs dão suporte aos residentes em medicina geral que trabalham nos Policlínicos, realizando, ainda, interconsultas, atividades docentes e investigações de saúde nos CMFs, visando à integração entre o trabalho assistencial e a docência, princípio básico de articulação de todo o sistema. Os GBTs se constituem considerando a área jurisdicional de um Policlínico ou Hospital Rural e, em algumas localidades, também contam com um técnico em estatística e um técnico em higiene e epidemiologia.

\section{Relação Público-Privado}

Conforme o art. 50 da Constituição da República de Cuba, proclamada em 1976, o direito à saúde é garantido pelo Estado, o que é efetivado de maneira totalmente pública e estatal. Não existe, portanto, participação da iniciativa privada no que se refere à prestação de serviços de saúde em Cuba.

\section{Cobertura a Medicamentos}

O Sistema Nacional de Medicamentos é integrante público e estatal do SNS, que tem por objetivo garantir o acesso - assistencial, econômico e geográfico - de toda a população aos medicamentos de que necessita, estando estes à disposição na rede de farmácias do país, com um preço fixado pelo Estado. A prescrição de medicamentos se rege pelas regulações do Programa Nacional de Medicamentos e pela 
estratégia da Farmácia Principal Municipal, direcionada aos médicos da atenção médica primária. A Farmácia Principal Municipal é a unidade gestora da Rede Municipal de Farmácias, constituída por farmácias comunitárias e farmácias vinculadas aos Policlínicos. $\mathrm{Na}$ atenção hospitalar e nos programas de saúde priorizados na atenção ambulatorial (HIV-AIDS, tuberculose, transplante de órgãos, entre outros), os medicamentos são distribuídos gratuitamente (Soberats et al., 2011).

\section{Discussões e Considerações Finais}

No presente trabalho, foram apresentados os sistemas de saúde do Brasil, do Canadá e de Cuba com base em elementos de análise determinados, sendo que alguns aspectos foram um pouco mais expostos do que outros em função dos parâmetros adotados e da disponibilidade de informações. Entende-se que a extensão das referências cubana e canadense na constituição do sistema de saúde do Brasil teve e tem impacto direto na efetividade das proposições da atenção primária em saúde e da Estratégia Saúde da Família, tal como estabelecidas em nosso país. A seguir, serão tecidas algumas reflexões acerca dos três sistemas de saúde, a fim de se ressaltar semelhanças, diferenças e possíveis influências sobre o sistema brasileiro, embora, não se descarte, obviamente, as influências dos sistemas de saúde de outros países que não foram analisados no recorte aqui apresentado.

$$
\text { Comparando-se as principais }
$$

características dos sistemas de saúde do Brasil, do Canadá (Quebec) e de Cuba, observa-se que estes se constituíram como sistemas públicos que atribuíram grande importância à atenção primária em saúde e estruturaram seus serviços em torno do cuidado às famílias. Indiferentemente se orientados por preceitos estabelecidos no Relatório Lalonde (1974/1981), publicado pelo Ministério da Saúde do Canadá, ou pela Declaração de Alma-Ata, editada pela OMS em 1978, bastante influente no Brasil e em Cuba, nota-se que cada país, quando analisado isoladamente, atentou-se para que seus respectivos sistemas tivessem a promoção da saúde como fundamento, levando $\mathrm{em}$ consideração as ponderações das análises efetuadas por Rabelo (2006; 2010). Entretanto, como cada país se apropriou desse conceito à sua maneira, podem ser notadas discrepâncias entre os três sistemas de saúde e, em especial no que se refere ao sistema de saúde canadense, são percebidas amplas diferenças, de ordem prática, em relação aos sistemas brasileiro e cubano, as quais serão descritas adiante.

Quanto aos princípios dos sistemas de saúde, percebe-se que muitos, guardadas algumas particularidades, podem ser reconhecidos como semelhantes. Nesse sentido, destacam-se os princípios da universalidade e da equidade (denominado como acessibilidade, para os canadenses), que estabelecem a cobertura dos serviços de saúde a todos os cidadãos, em condições uniformes e sob nenhuma forma de discriminação. Embora o sistema de saúde brasileiro seja reconhecidamente público, como trata a Constituição Federal, os princípios expressos na Lei $\mathrm{n}^{\circ} 8.080 / 90$ não trazem, 
explicitamente, a administração pública do sistema, enquanto Canadá e Cuba o fazem de forma clara, apresentando o caráter público do sistema como o primeiro princípio a ser cumprido. A garantia de atendimento integral, em todos os níveis de atenção, é assegurada nos princípios constitucionais dos sistemas do Brasil e de Cuba, ao passo que no sistema canadense o princípio da abrangência garante o atendimento somente dos serviços considerados clinicamente necessários. O princípio da descentralização é comum aos sistemas brasileiro e canadense, embora, efetivamente, este ocorra de forma muito mais marcante e decisiva na organização dos serviços do Canadá. Apesar de não estar explicitamente nomeado entre os princípios do sistema cubano, reconhece-se que, na prática, a descentralização direciona a organização do sistema de saúde no país. Destaca-se, ainda, a participação popular, relevante entre os princípios dos sistemas de saúde do Brasil e de Cuba, e regidos por legislação específica nos dois países, mas que não tem equivalente entre os princípios canadenses. É importante ressaltar que alguns princípios são bastante específicos de cada sistema de saúde, como o princípio da transferibilidade/portabilidade canadense e o princípio do internacionalismo cubano.

$\mathrm{Na}$ organização dos sistemas de saúde, o papel atribuído ao médico de família como o de quem controla o acesso aos especialistas e às admissões hospitalares é similar nos três países. Por outro lado, a forma de estruturação do sistema de saúde do Canadá, bem como a cobertura dos serviços de saúde (que não é totalmente pública), a autonomia conferida à classe médica e a forma de remuneração desta são particularidades do sistema canadense. Cabe destacar, ainda, o fato de o serviço odontológico ser considerado suplementar no sistema de saúde do Canadá, enquanto no Brasil e em Cuba este constitui um aspecto importante do atendimento a ser prestado às famílias. Além disso, o fato de o Canadá ter treze sistemas de saúde (dez provinciais e três territoriais) confere uma diferença fundamental entre o sistema canadense e os sistemas cubano e brasileiro, caracterizados por serem sistemas únicos. No que se refere à organização dos sistemas de saúde, podem ser percebidas, portanto, muitas similaridades entre os sistemas de saúde brasileiro e cubano, especialmente em relação ao reconhecimento oficial da saúde como um direito de todos e um dever do Estado. Destacase também a organização da atenção médica nos níveis primário, secundário e terciário, e a estruturação similar da Equipe Básica de Saúde, com a diferença de que, no Brasil, conta-se com o Agente Comunitário de Saúde, enquanto em Cuba o médico de família é quem faz o contato mais próximo com a população. Além disso, percebem-se afinidades entre os Núcleos de Apoio de Saúde da Família brasileiros e os Grupos Básicos de Trabalho cubanos, bem como é semelhante a importância conferida à territorialidade. $\mathrm{O}$ espaço atribuído à participação popular na organização dos sistemas também é significativo nos sistemas do Brasil e de Cuba. Entretanto, uma diferença substancial entre esses dois sistemas reside no fato de que, no Brasil, a responsabilidade pela formação dos profissionais da área da saúde 
cabe ao Ministério da Educação, enquanto em Cuba essa função pertence ao Ministério de Saúde Pública, o que produz efeitos na formação prática dos estudantes.

A relação público-privado, ou seja, a participação da iniciativa privada na prestação de serviços de saúde difere significativamente entre os três países. Enquanto em Cuba os serviços de saúde são oferecidos de forma totalmente pública, no Brasil os mesmos serviços podem ser ofertados tanto pela iniciativa pública quanto privada; no Canadá, entretanto, é atribuída uma função bem mais restrita que no Brasil aos planos de saúde privados, uma vez que estes só podem concorrer na faixa de serviços não cobertos pelo governo. No caso brasileiro, como assinala Gerschman (2008), muitos arranjos de financiamento e provisão de serviços de saúde foram sugeridos ao longo da história, a fim de conciliar saúde pública e privada, o que originou um sistema de saúde bastante fragmentado. Tendo em vista que o setor privado tem crescido em sobreposição aos serviços prestados pelo SUS, essa coexistência de seguros públicos e privados para os mesmos serviços caracteriza-se num problema para o usuário brasileiro, que, muitas vezes, paga por um serviço coberto pelo sistema público. Observa-se que a relação público-privado distancia os sistemas de saúde canadense, cubano e brasileiro, não sendo possível determinar influências do Canadá e de Cuba sobre o sistema brasileiro.

Por fim, a cobertura a medicamentos guarda semelhanças e divergências particulares de cada caso analisado. $\mathrm{O}$ seguro público aos medicamentos empregados na atenção hospitalar é praticado da mesma forma nos três países. Já a cobertura dos medicamentos utilizados fora desse contexto é semelhante no Brasil e em Cuba, tendo em vista que alguns tipos são fornecidos gratuitamente, enquanto outros são subsidiados, apesar de, no Brasil, essa cobertura ser mais ampla por abranger todos os níveis de atenção. Nesse âmbito, o Governo Federal do Canadá não cobre os custos com medicamentos da maioria da população, deixando estes a cargo das províncias/territórios, os quais variam amplamente a extensão da cobertura.

Por meio deste panorama, em que foram comparados os sistemas de saúde brasileiro, canadense e cubano, avalia-se que, apesar de constantemente citada como referência em muitos textos acadêmicos, a influência canadense sobre o sistema brasileiro, na prática, é bastante limitada. Acredita-se que essa influência pode estar mais concentrada na incorporação de princípios básicos de promoção da saúde nas diretrizes brasileiras de atenção primária em saúde do que no modo concreto de estruturação dos serviços, o que deve motivar estudos posteriores que avaliem melhor tal aspecto.

No que se refere ao sistema de saúde brasileiro, observa-se que, entre os sistemas de saúde do Canadá e de Cuba, existe uma maior semelhança e afinidade com os princípios constitucionais, a organização da atenção à saúde da família e a cobertura a medicamentos, praticados no sistema cubano. Considera-se que a transposição de modelos assistenciais é permeada por circunstâncias diversas e, por isso, 
não se pode traçar uma relação de influência direta e linear entre os sistemas de Cuba e do Brasil. Além disso, um vasto conjunto de propostas influenciou as orientações políticas que embasaram teoricamente o sistema brasileiro, concomitantemente à referência cubana na época. Entretanto, quanto aos parâmetros analisados e em comparação ao que é executado no Canadá, observa-se que as características gerais do sistema de saúde de Cuba, esse pequeno país socialista latinoamericano, estiveram mais presentes, em termos estruturais e legais, na elaboração das diretrizes de saúde do Brasil, país capitalista de extensão continental.

\section{Referências}

Aguiar, D. S. (1998). A "saúde da família" no sistema único de saúde: um novo paradigma?. Dissertação de Mestrado, Escola Nacional de Saúde Pública, Fundação Oswaldo Cruz, Rio de Janeiro.

Albuquerque, F. J. B., \& Melo, C. F. (2010). Avaliação dos serviços públicos de saúde em duas capitais nordestinas do Brasil. Psicologia: Teoria e Pesquisa, 26(2), 323-330.

Bonet, O. (2014). Os médicos da pessoa: um olhar antropológico sobre a medicina de família no Brasil e na Argentina. Rio de Janeiro: 7Letras.

Conill, E. M. (2000). A recente reforma dos serviços de saúde na província do Québec, Canadá: as fronteiras da preservação de um sistema público. Cad. Saúde Pública, 16(4), 963-971.

Conill, E. M. (2007). Sistemas Comparados de
Saúde. In G. W. S. Campos, M. C. S. Minayo, M. Akerman, M. Drumond Junior \& Y. M. Carvalho (Orgs.). Tratado de Saúde Coletiva (pp. 563-613). São Paulo/Rio de Janeiro: Hucitec/Fiocruz.

Conill, E. M. (2008). Ensaio histórico-conceitual sobre a Atenção Primária à Saúde: desafios para a organização de serviços básicos e da Estratégia Saúde da Família em centros urbanos no Brasil. Cad. Saúde Pública, 24(Sup 1), S7-S27.

Conill, E. M. (2009). Os pontos positivos do sistema de saúde público canadense. Revista Ser Médico, 49, 13-15. Recuperado em 11 agosto, 2011, de http://www.cremesp.org.br/?siteAcao $=\mathrm{R}$ evista\&id $=445$

Conill, E. M. (2011). A Análise Comparada na Avaliação de Serviços e Sistemas de Saúde. In R. O. Campos \& J. P. Furtado (Orgs.). Desafios da Avaliação de Programas e Serviços em Saúde. Campinas/SP: Unicamp.

Conill, E. M., Mendonça, M. H., Silva, R. A. P. R., \& Gawryszewski, V. (1991). Organização dos serviços de saúde: a comparação como contribuição. Cadernos de Saúde Pública, 7(3), 328-346.

Constituição da República Federativa do Brasil. (1998, 05 de outubro). Recuperado em 03 outubro, 2011, de http://www.senado.gov.br/sf/legislacao/ const/

Constitución de la Republica de Cuba. (1976, 24 de fevereiro). Recuperado em 12 maio, 2012, de http://www.cuba.cu/gobierno/cuba.htm 
Costa, A. M., Pontes, A. C. R., \& Rocha, D. G. (2006). Intersetorialidade na produção e promoção da saúde. In A. Castro \& M. Malo (Orgs.). SUS: ressignificando a promoção da saúde (pp. 96-115). São Paulo: Hucitec.

Decreto n ${ }^{\circ}$ 5.090. (2004, 20 de maio). Regulamenta a Lei $\mathrm{n}^{\circ}$ 10.858, de 13 de abril de 2004, e institui o programa "Farmácia Popular do Brasil” e dá outras providências. Brasília, DF: Presidência da República.

Gerschman, S. (2008). Políticas comparadas de saúde suplementar no contexto de sistemas públicos de saúde: União Europeia e Brasil. Ciência \& Saúde Coletiva, 13(5), 1441-1451.

Gil, A. C. (1999). Métodos e técnicas de pesquisa social (5a ed.). São Paulo: Atlas.

Gouvernement du Québec. (2008). O sistema de saúde e serviços sociais no Quebec. Quebec: Autor.

Health System and Policy Division (1999). O sistema de cuidados de saúde do Canadá. Ottawa: Autor.

Hortale, V. A., Conill, E. M., \& Pedroza, M. (1999). Desafios na construção de um modelo para análise comparada da organização de serviços de saúde. Cad. Saúde Pública, 15(1), 79-88.

Labra, M. E. (2001). Política e saúde no Chile e no Brasil. Contribuições para uma comparação. Ciência \& Saúde Coletiva, 6(2), 361-376.

Lalonde, M. (1981). A new perceptive on the health of Canadians: a working document. Ottawa: Ministry of Health and Welfare. (Original publicado em 1974).
Lei $n^{0}$ 8.080. (1990, 19 de setembro). Dispõe sobre as condições para a promoção, proteção e recuperação da saúde, a organização do funcionamento dos serviços correspondentes e dá outras providências. Brasília, DF: Diário Oficial da União.

Lei no 9.565. (1998, 2 de junho). Cria a Agência Nacional de Saúde e dá outras providências. Brasília, DF: Diário Oficial da União.

Lei $n^{0}$ 12.871. (2013, 22 de outubro). Institui o Programa Mais Médicos, altera as Leis $\mathrm{n}^{\circ}$ 8.745, de 9 de dezembro de 1993, e $\mathrm{n}^{\circ}$ 6.932, de 7 de julho de 1981, e dá outras providências. Brasília, DF: Diário Oficial da União.

Marchildon, G. P. (2013). Canada: examen du système de santé. In Systèmes de Santé en Transition, 15(1), 1-183.

Ministério da Saúde. Secretaria de Políticas de Saúde. Departamento de Atenção Básica. (2001). Política Nacional de Medicamentos do Brasil. Brasília, DF: Autor.

Organização Mundial da Saúde (2015). Dados estatísticos. Recuperado em 23 dezembro, 2016, de http://www.who.int/countries/en/

Organização Pan-Americana de Saúde (2007). As políticas e os sistemas e serviços. In Autor, Saúde nas Américas (Vol. 1, pp. 314405). Brasília: Organização PanAmericana de Saúde.

Portaria $n^{\circ}$ 648. (2006, 28 de março). Aprova a Política Nacional de Atenção Básica, estabelecendo a revisão de diretrizes e 
normas para a organização da Atenção Básica para o Programa Saúde da Família (PSF) e o Programa Agentes Comunitários de Saúde (PACS). Brasília, DF: Ministério da Saúde.

Portaria $n^{\circ}$ 2.488. (2011, 21 de outubro). Aprova a Política Nacional de Atenção Básica, estabelecendo a revisão de diretrizes e normas para a organização da Atenção Básica, para a Estratégia Saúde da Família (ESF) e o Programa Agentes Comunitários de Saúde (PACS). Brasília, DF: Ministério da Saúde.

Portaria $n^{\circ}$ 111. (2016, 28 de janeiro). Dispõe sobre o Programa Farmácia Popular do Brasil (PFPB). Brasília, DF: Ministério da Saúde.

Rabelo, L. S. (2006). Promoção da Saúde: desafio ou adaptação? A construção do conceito, de Alma-Ata aos dias atuais, no Brasil e no Canadá. Tese de Doutorado, Instituto de Ciências Sociais, Universidade de Brasília, Brasília.

Rabelo, L. S. (2010). Estudo Comparado Brasil e Canadá. In Promoção da Saúde: a construção social de um conceito em perspectiva comparada (pp. 107-155). Rio de Janeiro: Fiocruz.

Rêgo, E. C. L. (2000). Políticas de regulação do mercado de medicamentos: a experiência internacional. Revista do BNDES, 7(14), 367-400.

Santos, A. M. S. P., \& Ugá, M. A. D. (2007). Reformas do Estado, descentralização e políticas de saúde: uma análise comparada entre Argentina, Brasil, Colômbia e México. Ensaios FEE, 28(2), 525-548.
Silveira, C. H. (2008). Falando de modelos de assistência à saúde e do programa de saúde da família do Ministério da Saúde - Brasil. R. Inter. Interdisc. INTER thesis, 5(1), 78-103. Soberats, F. S., Márquez, M., \& Galbán, P. A. (2011). Medicina general, medicina familiar: experiencia internacional y enfoque cubano. Havana: Editorial Ciencias Médicas.

Sório, R. S. R. (2006). O sistema de saúde canadense e as reformas em curso. In G. Biasoto Júnior, P. L. B. Silva \& S. Dain (Orgs). Regulação do setor saúde nas Américas: as relações entre o público e o privado numa abordagem sistêmica (pp. 143-177). Brasília: Organização Pan-Americana de Saúde.

Viana, A. L. D., \& Dal Poz, M. R. (2005). A reforma do sistema de saúde no Brasil e o Programa de Saúde da Família. Physis: Revista de Saúde Coletiva, 15(Suplemento), 225-264.

Recebido em 16/01/2017

Aprovado em 05/06/2017 